\title{
Effect of Social Facilitation without Presence of Others on Swallowing Function and Eating Behavior
}

\author{
Kengo Hoyano ${ }^{1,2 *}$ and Yasuhiro Ogoshi ${ }^{3}$ \\ ${ }^{1}$ Department of Human and Artificial Intelligent Systems, Graduate School, University of Fukui, \\ 3-9-1 Bunkyo, Fukui 910-8507, Japan \\ ${ }^{2}$ Department of Rehabilitation Speech-Language-Hearing Therapy, Fukui College of Health Sciences, \\ 55 Egami-cho 13-1, Fukui 910-3190, Japan \\ ${ }^{3}$ Graduate School of Engineering, University of Fukui, \\ 3-9-1 Bunkyo, Fukui 910-8507, Japan
}

(Received January 17, 2018; accepted May 7, 2018)

Keywords: social facilitation, eating behavior, swallowing function, diet modification for dysphagia

Humans have an increased sense of taste and consume more food when eating together with others. This effect is called the social facilitation of eating. In recent years, social facilitation of eating has been reported to occur even without the presence of others. In this study, we examined whether social facilitation of eating can be induced when consuming a diet modified for dysphagia. Using a monitor, we set two conditions: a person-reflecting condition and a wallreflecting condition. Participants were given a gelled liquid of green tea or orange juice under each condition. The results showed that under the person-reflecting condition, the consumption of the gelled liquid was significantly larger, and participants evaluated that it was significantly easier to swallow and that the taste was significantly better. From these results, it is thought that social facilitation of eating occurred even with a diet modified for dysphagia. Because the effect was obtained using only an image captured by a web camera, it could be applied to techniques such as virtual reality (VR). The possibility of using this effect for the rehabilitation of dysphagia patients was demonstrated. In future work, we will construct a system to support the rehabilitation of dysphagia patients and provide meals with community-dwelling adults using a head-mounted display (HMD).

\section{Introduction}

Many factors are involved in human eating behavior. The surrounding environment is one such factor. The part of the surrounding environment shown to affect eating behavior is whether people eat together. ${ }^{(1)}$ Previous studies have reported that intake during meals increases when eating with other people. ${ }^{(2)}$ de Castro reported that when participants ate with someone, food intake increased by 40 to $50 \%$ on average. ${ }^{(3)}$ Lumeng and Hillman reported that infants also have an increased food intake when the number of people in a group is large. ${ }^{(4)}$ Berry et al. reported an investigation involving ice cream, in which they found that individuals ingested

*Corresponding author: e-mail: fcm-k.hoyano@kzh.biglobe.ne.jp

This was published on July 13, 2018, and editorial errors were corrected on July 17, 2018.

http://dx.doi.org/10.18494/SAM.2018.1912 
greater amounts of ice cream in a small group than when they were alone. ${ }^{(5)}$ In these reports, social facilitation is cited as the reason behind the increased food intake. Social facilitation is said to be a phenomenon in which certain behaviors of individuals are promoted by the presence of others. ${ }^{(6,7)}$ This promotion of behavior has been observed during eating and is called social facilitation of eating. ${ }^{(8-11)}$ Social facilitation of eating usually requires the presence of others. As an example, when chocolate was eaten together with strangers, it was determined to be richer and more flavorful than when eaten alone. ${ }^{(12)}$ Several reports suggest that the presence of others is necessary for this effect to occur but, in recent years, some contrary results have been reported. Nakata and Kawai conducted an experiment in which participants ate as much popcorn as they liked, under a condition where their image was reflected on a monitor or where the wall was reflected on a monitor. The results showed that both the amount of popcorn consumed and the flavor rating of popcorn were higher when the popcorn was eaten with the image of the participant displayed in the mirror. ${ }^{(13)}$ From this, it is considered that the presence of others is not necessary for social facilitation of eating. In daily life, it is common to share meals with others. However, in environments such as hospitals, it is often difficult to eat with others. In addition, patients with dysphagia may have modified diets. The diet modification required for dysphagia, such as food prepared in the form of paste, is different from a normal diet. Therefore, many patients do not eat out because of the peculiarities of the diet. Patients that require diet modification for dysphagia are reported to have lower nutritional intake and muscle mass. ${ }^{(14)}$ It can be seen that if social facilitation of eating is also demonstrated with diets modified for dysphagia, even in a solitary situation, the amount of food intake can be increased and the image of diet modification for dysphagia can be changed. It is also thought that this may aid in the recovery of patients. From this point of view, we examined whether social facilitation of eating influences the intake of a diet modification for dysphagia and the possibility of application to a support system using a head-mounted display (HMD).

\section{Materials and Methods}

\subsection{Participants}

We enlisted eight young adults (four females and four males, aged 20-21 years with a mean of 20.63 years) who were healthy and who had no history of special dietary restrictions, food allergies, or eating disorders.

\subsection{Procedure}

The participants were experimented $5 \mathrm{~h}$ after ingesting lunch. They were instructed to evaluate the taste of various gelled liquids and to eat as much gelatinous liquid as they wanted. They were asked to sit on a chair in front of a desk. A 22-inch LED monitor (Dell Co., Ltd.; P2213) was placed on the desk, and a web camera (Elecom Co., Ltd.; UCAM-C 0220 FBNBK) was installed on the monitor. The distance from the participants to the monitor was about 50 $\mathrm{cm}$. There were two conditions: an image of the participant was displayed on the monitor using the web camera (person-reflecting condition), or the wall behind the monitor was displayed 
on the monitor using the web camera (wall-reflecting condition). Under the person-reflecting condition, the web camera was adjusted so that the upper body of the subject appeared on the monitor.

Two kinds of gelled liquids were presented to participants: green tea (Suntory Co., Ltd.; iemon) and orange juice (Asahi Soft Drink Co., Ltd.; Bireley's Orange). The reason for using green tea is that it is often taken by patients with dysphagia. Orange juice was used as a beverage reflecting sweetness because it is considered that using only green tea could participants biased preference.

A 3 g quantity of a gelling agent (Healthy Food Co., Ltd.; Toromi Power Smile) was used to produce gels of the two kinds of liquids (100 ml each). Each gelled liquid was placed in a plastic container. A stainless-steel spoon $(5 \mathrm{ml})$ was used to eat the gel. The participants ate one type of gelled liquid at a time. They were presented with four $(2 \times 2)$ different conditions (person-reflecting or wall-reflecting condition and green tea or orange juice) in a randomized order.

The participants entered the laboratory and sat on a chair. They were handed a laboratory vessel containing a gelled liquid and a spoon. They were instructed to take as much gelled liquid as desired until the alarm sounded. They were not instructed to direct their attention to the monitor. After the experimenter's cue, the participants started to ingest the gelled liquid by themselves. The ingestion period lasted $90 \mathrm{~s}$. The end of the ingestion period was signaled by an alarm, and the participants then answered a questionnaire to subjectively evaluate the gelled liquid. Subsequently, the participants started the experiment again under the next combination of conditions. Plastic containers containing a gelled liquid and a stainless-steel spoon were handed over each time the conditions changed.

We used a questionnaire to examine the influence of social facilitation on subjective evaluation at eating. A visual analog scale (VAS) was used for the subjective evaluation questionnaire. A line segment of $10 \mathrm{~cm}$ length was set for each question regarding the participant's subjective evaluation of the gelled liquid. The left side of the line was labelled "very" and the right side was labelled "not at all". The participants answered each question by marking the line segments. The subjective evaluation was measured by the distance $(\mathrm{cm})$ from the left end of the line segment to the mark (i.e., $0 \mathrm{~cm}=$ "very", $10 \mathrm{~cm}=$ "not at all"). The questionnaire included items such as "easiness of swallowing" reflecting the swallowing function, "desirability" reflecting the impression on food, and "taste" reflecting tastes like "deliciousness", "sweetness", or "bitterness". Each question was written in Japanese. The questions were as follows: "How easy is it to swallow this liquid?", "How much do you like this liquid?", "How delicious is this liquid?", "How sweet is this liquid?", and "How bitter is this liquid?". We also calculated the consumption ratio of each bowl of gelled liquid by subtracting the amount of remaining gelled liquid from the initial amount (i.e., 100\%).

\subsection{Statistical analysis}

A paired $t$-test was carried out on consumption rate and subjective evaluation by VAS. The statistical significance threshold was set at less than 5\%. Statistical assessments were conducted using SPSS Statistics version 25 (IBM Co., Ltd.). ${ }^{(15)}$ 


\section{Results}

\subsection{Consumption ratio}

The green tea consumption ratio was $15.63 \%$ [standard error $(\mathrm{SE})=2.23$ ] under the personreflecting condition and 9.75\% $(\mathrm{SE}=1.78)$ under the wall-reflecting condition $[t(7)=3.707, p=$ 0.007]. The orange juice consumption ratio was $47.38 \%(\mathrm{SE}=7.75)$ under the person-reflecting condition and $31.25 \%(\mathrm{SE}=6.48)$ under the wall-reflecting condition $[t(7)=3.62, p=0.008]$ (Fig. 1).

\subsection{Subjective evaluations of diet}

The evaluation of easiness of swallowing was as follows: for green tea, the combined VAS score was $5.91 \mathrm{~cm}(\mathrm{SE}=0.76)$ under the person-reflecting condition and $7.29 \mathrm{~cm}(\mathrm{SE}=1.16)$ under the wall-reflecting condition $[t(7)=-2.517, p=0.04]$; for orange juice, it was $4.24 \mathrm{~cm}$ (SE $=0.98)$ under the person-reflecting condition and $5.41 \mathrm{~cm}(\mathrm{SE}=0.83)$ under the wall-reflecting condition $[t(7)=-2.434, p=0.045]$ (Fig. 2).

The evaluation of taste was as follows: for green tea, the combined VAS score was $7.9 \mathrm{~cm}$ (SE $=0.54)$ under the person-reflecting condition and $9.58 \mathrm{~cm}(\mathrm{SE}=0.11)$ under the wall-reflecting condition $[t(7)=-3.6, p=0.008]$; for orange juice, it was $1.39 \mathrm{~cm}(\mathrm{SE}=0.49)$ under the personreflecting condition and $2.94 \mathrm{~cm}(\mathrm{SE}=0.57)$ under the wall-reflecting condition $[t(7)=-3.31, p$ $=0.012]$ (Fig. 3).

The evaluation of the likeability was as follows: for green tea, the combined VAS score was 8.78 $\mathrm{cm}(\mathrm{SE}=0.51)$ under the person-reflecting condition and $8.67 \mathrm{~cm}(\mathrm{SE}=0.40)$ under the wallreflecting condition $[t(7)=0.284, p=0.785]$; for orange juice, it was $2.08 \mathrm{~cm}(\mathrm{SE}=0.68)$ under the person-reflecting condition and $2.58 \mathrm{~cm}(\mathrm{SE}=0.54)$ under the wall-reflecting condition $[t(7)$ $=-1.186, p=0.274$ ] (Fig. 4).

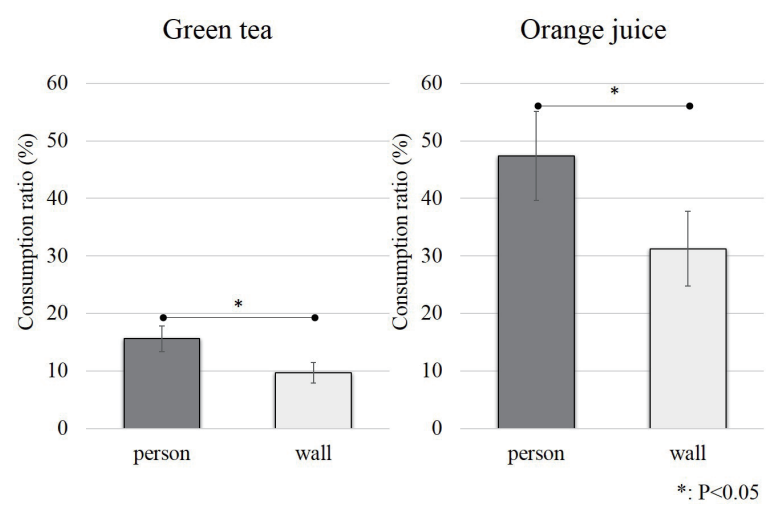

Fig. 1. Consumption ratio. Average $\pm \mathrm{SE}$.

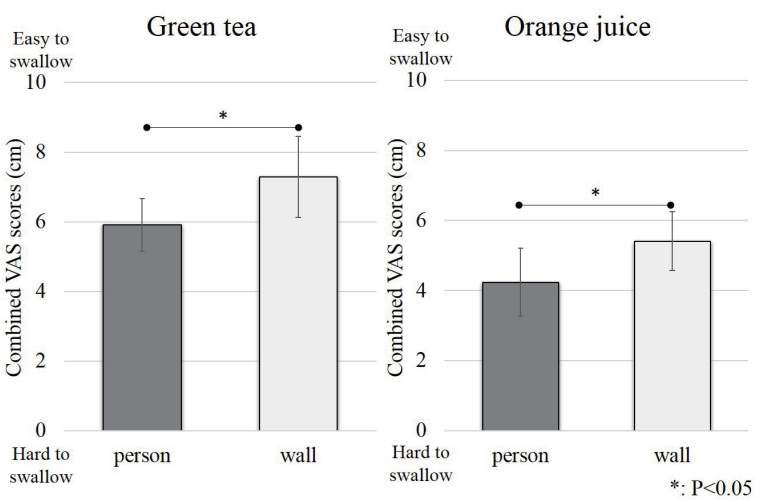

Fig. 2. Evaluation of ease of swallowing (combined VAS scores). Smaller numbers indicate easier to swallow. Average $\pm \mathrm{SE}$. 


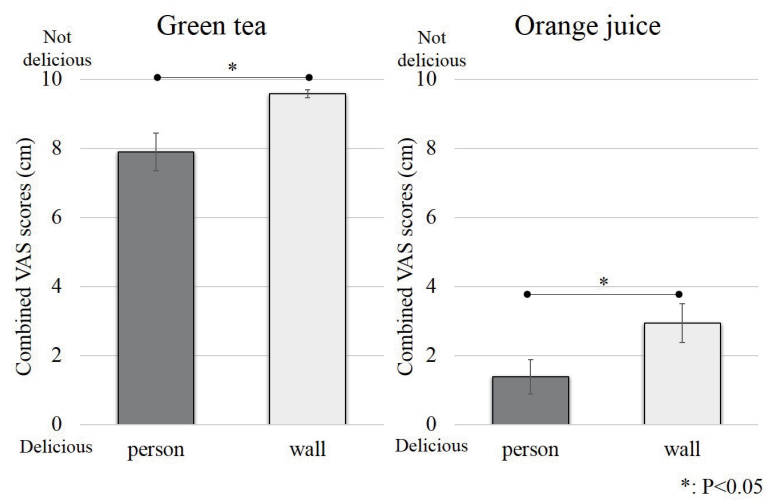

Fig. 3. Evaluation of deliciousness (combined VAS scores). Smaller numbers indicate delicious. Average \pm SE.

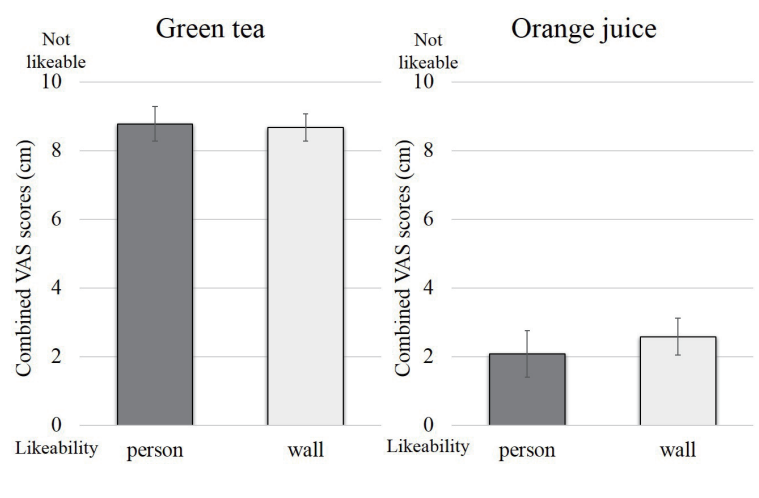

Fig. 4. Evaluation of likeability (combined VAS scores). Smaller numbers indicate likeability. Average \pm SE.

The evaluation of the sweetness was as follows: for green tea, the combined VAS score was 9.54 $\mathrm{cm}(\mathrm{SE}=0.15)$ under the person-reflecting condition and $9.58 \mathrm{~cm}(\mathrm{SE}=0.12)$ under the wallreflecting condition $[t(7)=-0.202, p=0.846]$; for orange juice, it was $1.14 \mathrm{~cm}(\mathrm{SE}=0.26)$ under the person-reflecting condition and $2.23 \mathrm{~cm}(\mathrm{SE}=0.32)$ under the wall-reflecting condition $[t(7)$ $=-4.856, p=0.002]$ (Fig. 5).

The evaluation of the bitterness was as follows: for green tea, the combined VAS score was 4.49 $\mathrm{cm}(\mathrm{SE}=1.03)$ under the person-reflecting condition and $3.60 \mathrm{~cm}(\mathrm{SE}=0.89)$ under the wallreflecting condition $[t(7)=3.253, p=0.014]$; for orange juice, it was $9.74 \mathrm{~cm}(\mathrm{SE}=0.09)$ under the person-reflecting condition and $9.59 \mathrm{~cm}(\mathrm{SE}=0.22)$ under the wall-reflecting condition $[t(7)$ $=0.716, p=0.497]$ (Fig. 6).

\section{Discussion}

The consumption ratio was significantly higher under the person-reflecting condition than under the wall-reflecting condition for both green tea and orange juice. Previous studies revealed similar results. ${ }^{(13)}$ This study revealed that the consumption ratio increased with social facilitation. In previous studies, dietary intake increased owing to social facilitation. ${ }^{(2-5)}$ From these findings, it can be suggested that the consumption ratio increased because of social facilitation for a dysphagia-modified diet.

The VAS results for easiness of swallowing showed that both the green tea and the orange juice were significantly easier to swallow under the person-reflecting condition than under the wall-reflecting condition. It has been reported that exercise performance improves with reward. ${ }^{(16,17)}$ In terms of deliciousness, both the green tea and the orange juice had significantly better results under the person-reflecting condition than under the wall-reflecting condition. This deliciousness became a reward, and the subjective easiness of swallowing was improved by improving exercise performance. 


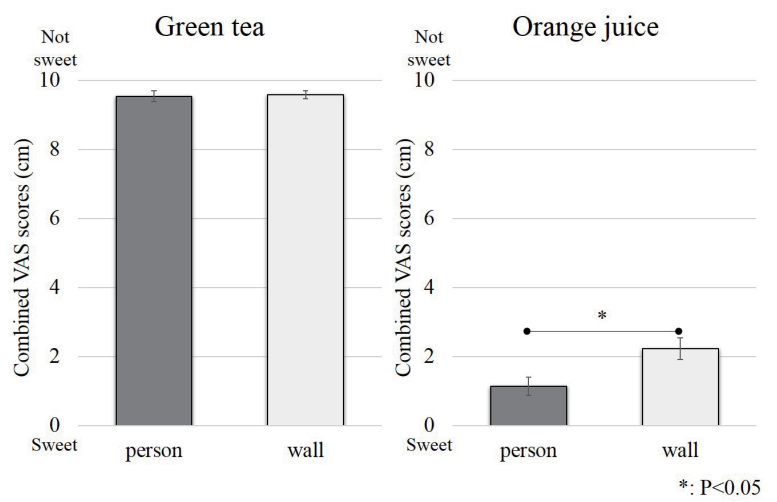

Fig. 5. Evaluation of sweetness (combined VAS scores). Smaller numbers indicate sweet. Average \pm SE.

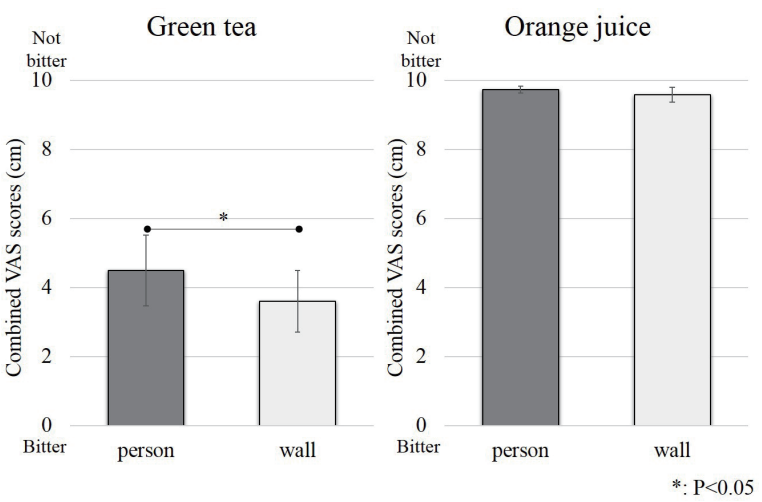

Fig. 6. Evaluation of bitterness (combined VAS scores). Smaller numbers indicate bitter. Average \pm SE.

Significant differences were found in deliciousness, sweetness, and bitterness. Both the green tea and the orange juice were rated as being significantly more delicious under the person-reflecting condition. Orange juice was rated as being significantly sweeter under the person-reflecting condition. Green tea was rated as being not significantly more bitter under the person-reflecting condition.

In terms of preference, there was no significant difference between green tea and orange juice. Other groups previously reported that information presented in advance affected food selectivity. ${ }^{(18-20)}$ It is thought that the prejudice that the food was a gelled liquid affected the results in that study.

From the results of this study, it was thought that social facilitation occurs even with a dysphagia-modified diet. Diet modification is required for patients whose swallowing function was deteriorated as a result of dysphagia. Food modified for dysphagia patients often has a different form, such as a paste, compared with ordinary food, which may cause changes in eating habits. For taste, the appearance of the food is also important. ${ }^{(21)}$ However, dysphagia may be directly linked to complications such as aspiration unless the form of the foods has been adjusted, and patients may require diet modification for dysphagia. Consequently, it is important that food modified for dysphagia be both delicious and safe. In this experiment, it was noteworthy that social facilitation occurred even with images captured by web cameras. Such a situation was effective not only in improving the perceived taste but also in improving the perceived easiness of swallowing; so it is conceivable that not only eating behavior but also swallowing function was affected. On the basis of this result, we suggest that the swallowing function and eating behavior of humans can be influenced by visual technology. Clinically, in addition to the possibility of enhancing the effect of rehabilitation for dysphagia, there is also the possibility that this technique may be effective in improving the nutritional status of patients with malnutrition or loss of appetite. In this study, there is a possibility that the null hypothesis may be rejected because the sample size is not sufficient. In the future, it is necessary to estimate the sample size being based on the results of this experiment and then to increase the 
number of participants in the following experiments. On the other hand, the consumption rate and taste of green tea and the sweetness of orange juice were considered to be highly accurate estimates because the SE is small.

Reinforcement learning is a type of motor learning. Reinforcement learning is a theory that suggests that learning behavior is selected to maximize reward. ${ }^{(22)}$ The cause of dysphagia is a motor dysfunction of the swallowing organ. Therefore, rehabilitation of dysphagia patients requires relearning the swallowing movement. Social facilitation makes food delicious to eaters, which is a reward. The effect of social facilitation may promote relearning of swallowing movements in the rehabilitation of dysphagia patients. In addition, it is possible to maintain or increase the amount of food consumed by hoping for a reward based on the deliciousness of the meal. Information that a reward is expected is conveyed to the brain and acts to improve behavioral performance to earn the reward. ${ }^{(23)}$ It has been reported that community-dwelling older adults have fewer kinds of foods to eat and may be malnourished. ${ }^{(24)}$ From these results, social facilitation may be able to prevent lack of diet and malnutrition of community-dwelling older adults. In future work, we will compare a person who eats with others and a meal environment created with virtual reality (VR) or augmented reality, construct a system to support the rehabilitation of dysphagia patients and provide meals with community-dwelling adults using an HMD, and verify the effect of this system. We will examine the training effect by measuring the activity of suprahyoid muscles during the rehabilitation of dysphagia patients using the HMD system with an electromyogram or by measuring the activity with an electroencephalograph.

\section{Conclusions}

In this study, the social facilitation of eating was observed when ingesting food modified for dysphagia. We also found that even when an individual's own image was displayed using a web camera, social facilitation of eating occurred. The results of this study demonstrate that a visual information system, such as VR, could be constructed and used for the rehabilitation of dysphagia patients. We are considering application to a support system for patients with dysphagia and community-dwelling adults using an HMD.

\section{Acknowledgments}

The authors express their gratitude to the students of Fukui College of Health Sciences who kindly offered their cooperation as participants of this study despite the substantial time commitment.

\section{References}

1 S. Imada: The Psychology of Eating: Why We Eat What We Eat (Yuhikaku, Tokyo, 2005) p. 6 (in Japanese).

2 C. P. Herman, D. A. Roth, and J. Polivy: Psychol. Bull. 129 (2003) 873.

3 J. M. de Castro: Physiol. Behav. 47 (1990) 1129.

4 J. C. Lumeng and K. H. Hillman: Arch. Dis. Child. 92 (2007) 384. 
5 S. L. Berry, W. W. Beatty, and R. C. Klesges: Appetite 6 (1985) 41.

6 M. Isozaki: Jpn. J. Exp. Soc. Psychol. 19 (1979) 49.

7 F. H. Allport: J. Exp. Psychol. 3 (1920) 159.

8 J. M. de Castro, J. McCormick, M. Pedersen, and S. N. Kreitzman: Physiol. Behav. 38 (1986) 25.

9 B. Wansink and S. B. Park: Food Qual. Prefer. 12 (2001) 69.

10 V. I. Clendenen, C. P. Herman, and J. Polivy: Appetite 23 (1994) 1.

11 S. C. King, H. L. Meiselman, A. W. Hottenstein, T. M. Work, and V. Cronk: Food Qual. Prefer. 18 (2007) 58.

12 E. J. Boothby, M. S. Clark, and J. A. Bargh: Psychol. Sci. 25 (2014) 2209.

13 R. Nakata and N. Kawai: Physiol. Behav. 179 (2017) 23.

14 A. Shimizu, K. Maeda, K. Tanaka, M. Ogawa, and J. Kayashita: Geriatr. Gerontol. Int. 18 (2017) 698. doi: 10.1111 /ggi.13233

15 IBM SPSS: https://www.ibm.com/analytics/data-science/predictive-analytics/spss-statistical-software (accessed April 2018).

16 S. K. Sugawara, S. Tanaka, S. Okazaki, K. Watanabe, and N. Sadato: PLoS One 7 (2012) e48175.

17 B. H. Dobkin, P. Plummer-D'Amato, R. Elashoff, and J. Lee: Neurorehabil. Neural Repair 24 (2010) 235.

18 S. M. McClure, J. Li, K. S. Cypert, L. M. Montague, and P. R. Montague: Neuron 44 (2004) 379.

19 N. Sakai: J. Color Sci. Assoc. Jpn. 34 (2010) 343.

20 N. Sakai: ChemoSense 11 (2009) 1.

21 F. Egashira: J. Jpn. Soc. Parenter. Enter. Nutr. 31 (2016) 693.

22 K. Doya: Curr. Opin. Neurobiol. 10 (2000) 732.

23 N. Osaka: Social Brain Series Vol. 5: Brain Wired for Reward Expectancy: New Directions toward Neuroeconomics (Shinyosha, Tokyo, 2014) p. 133 (in Japanese).

24 Y. Kimura, T. Wada, K. Okumiya, Y. Ishimoto, E. Fukutomi, Y. Kasahara, W. Chen, R. Sakamoto, M. Fujisawa, K. Otsuka, and K. Matsubayashi: J. Nutr. Health Aging 16 (2012) 728.

\section{About the Authors}

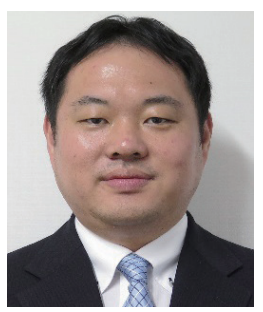

Kengo Hoyano is a research associate at the Department of Rehabilitation Speech-Language-Hearing Therapy, Fukui College of Health Sciences. He received an M.Sc. from the Department of Neurorehabilitation, Graduate School of Health Sciences, Kio University in 2014. He is engaged in studies relating to dysphagia and neuroscience.

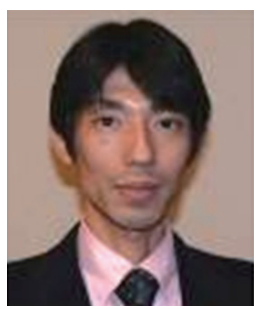

Yasuhiro Ogoshi is an associate professor at the Graduate School of Engineering, University of Fukui in Japan. He received a Ph.D. degree in engineering from the University of Kanazawa in 2001. He is engaged in studies related to human interfaces. 